\title{
Anterior Inferior Tibiofibular Ligament Reconstruction with Anchor Sutures compared with Trans-syndesmotic Screw Fixation for Ankle Syndesmotic Injuries
}

Jin Su Kim

\begin{abstract}
Background: Acute syndesmotic injuries were usually treated with trans-syndesmotic screw fixation. However, screw fixation is too strong compared with the physiologic ankle joint movement. In addition, it needs removal and leads to delayed rehabilitation. We propose the reconstruction of the anterior inferior tibiofibular ligament (AITFL) using suture anchors to substitute trans-syndesmotic screw fixation.
\end{abstract}

Materials and methods: We compared the results after transsyndesmotic screw fixation and AITFL suture anchor fixation in syndesmotic injuries with or without ankle fracture. Consecutively, the trans-syndesmotic screw (group I) was conducted between June 2011 and June 2013, and since July 2013, suture anchor fixation was performed (group II). Reductions in quality, the American Orthopedic Foot and Ankle Society (AOFAS) score, and the Olerud-Molander ankle score (OMAS) were evaluated.

Results: The final AOFAS score and OMAS in both groups were not significantly different $(p=0.98,0.67)$. Tibiofibular overlapping, tibiofibular space, and tibiofibular overlapping ratio to the tibial width in both groups had satisfactory reduction from the standards. Computed tomographic (CT) evaluation also confirmed that both groups had been anatomically reduced in standard. Anteroposterior axis reduction in CT was more accurate in group II. Nonclinically related complications were three broken screws in final follow-up in group I.

Conclusion: Both trans-syndesmotic screws and suture anchor fixation have satisfactory clinical outcomes. The suture anchor fixation for syndesmotic injury does not need removal and is less complicated compared with the trans-syndesmotic screw fixation.

Keywords: Anchor, Ankle, Screw, Syndesmosis.

How to cite this article: Kim JS. Anterior Inferior Tibiofibular Ligament Reconstruction with Anchor Sutures compared with Trans-syndesmotic Screw Fixation for Ankle Syndesmotic Injuries. J Foot Ankle Surg (Asia-Pacific) 2017;4(2):57-62.

Source of support: Nil

Conflict of interest: None

Consultant

Foot and Ankle Surgery, CM General Hospital, Seoul, South Korea

Corresponding Author: Jin Su Kim, Consultant, Foot and Ankle Surgery, CM General Hospital, Seoul, South Korea, Phone: 821045987459, e-mail: jins33@hanmail.net

\section{INTRODUCTION}

Distal syndesmosis is known to be important for the function and stability of the ankle joint. ${ }^{1}$ Distal syndesmotic injuries often occur due to pronation-external rotation or pronation-eversion injury. ${ }^{2-4}$ If the acute unstable syndesmotic injury is not treated appropriately, chronic syndesmotic instability can develop, followed by osteoarthritis, which requires anatomical restoration. ${ }^{5,6}$ The trans-syndesmotic screw fixation is most widely used for distal syndesmotic injuries as an operative treatment. Trans-syndesmotic screw fixation provides firm fixation at syndesmosis; however, this method requires a removal process and can lead to screw failures along with high risk of inaccurate anatomical reduction. ${ }^{7,8}$ To overcome these drawbacks, a new fixation method involving the use of a suture button has been used. In previous studies, trans-syndesmotic suture-button fixation has been confirmed to have favorable results and stability, similar to the trans-syndesmotic screw fixation. ${ }^{8-10}$ Accordingly, the comparison study of several kinds of syndesmotic suture-button fixations has shown that suture-button fixation using conventional pathways shows vulnerability to the anterior and internal tractions, and to the external rotational force. ${ }^{3,11}$ The results of the biomechanical study have indicated that anatomical suture-button fixation from posterior cortex of fibular to anterolateral cortex of tibia provided dynamic stability and physiologic motion.

Recently, arthroscopic syndesmotic repair was introduced that used suture anchor from the anterior surface of fibular to distal surface of the distal tibia over the AITFL. ${ }^{12}$ This technique is a direct anatomical fixation for the anterior "open book" type syndesmotic injury. Another author has reported on the open AITFL reconstruction with the same concept using extensor digitorum longus of fourth toe for external rotation-type fracture with AITFL rupture. ${ }^{13}$ However, they have not conducted comparative studies.

The aim of the present study is to compare the clinical and radiological results of open anatomical reconstruction of AITFL using suture anchors with the conventional screw fixation method for syndesmotic injuries. 


\section{MATERIALS AND METHODS}

\section{Study Subjects}

The patients who enrolled between November 2011 and November 2014 to undergo operation due to AITFL injuries with or without fracture confirmed by ankle radiograph, magnetic resonance imaging (MRI), and intraoperative stress test were included in the study. Those who had a medical history of operation, structural deformation, or functional problems were excluded from this study. Institutional Review Board approval was obtained to conduct a retrospective design with prospective scoring. All patients gave their informed consent prior to the operation. Of the 64 screened patients, 26 patients who could not be followed up or who were finally followed up for clinical assessment were also excluded. Thirty-eight subjects were eventually selected. Between June 2011 and June 2013, trans-syndesmotic screw fixations were conducted (group I), and since July 2013, suture anchor fixations were performed (group II) consecutively. The mean age of the patients was $35.8(15-70)$ years, and the mean follow-up period was $24.2(12-44)$ months. Group I had 21 patients, and group II 25 (Table 1).

\section{Diagnosis of the AITFL Injuries}

If syndesmotic injury was suspected by squeeze test, external rotation test, and tenderness on AITFL and deltoid ligament, then ankle radiograph was checked. A diagnosis was made when two or three findings were observed-a $10 \mathrm{~mm}$ or less tibiofibular overlapping distance, a $5 \mathrm{~mm}$ or more tibiofibular space, and a $24 \%$ or less tibiofibular overlapping ratio to the tibial width. When the positive physical exam of syndesmotic injury was checked preoperatively, we checked ankle MRI scan including an AITFL oblique axial plan. ${ }^{14}$ If we found an

Table 1: Patient demographics

\begin{tabular}{lll}
\hline & $\begin{array}{l}\text { Trans- } \\
\text { syndesmotic } \\
\text { screw (group I) }\end{array}$ & $\begin{array}{l}\text { Suture } \\
\text { anchors } \\
\text { (group II) }\end{array}$ \\
\hline Patients (N) & 21 & 25 \\
Age (years) & $34.4(15-62)$ & $37.6(16-70)$ \\
Gender (male: female) & $13: 8$ & $13: 4$ \\
Follow-up (months) & $34.0(25-46)$ & $15.5(12-28)$ \\
Isolated injury & 1 & 3 \\
Associated fracture & 20 & 22 \\
Weber classification & & \\
$\quad$ Weber B & 14 & 16 \\
$\quad$ Weber C & 6 & 6 \\
Lauge-Hansen classification & & \\
Supination-external rotation II & 2 & 2 \\
Supination-external rotation IV & 12 & 14 \\
Pronation-external rotation III & 5 & 6 \\
Screw breakage & 3 & 0 \\
\hline
\end{tabular}

evidence of syndesmotic injury in MRI, we explored the AITFL injury with an anterolateral deep dissection. Finally, we confirmed with external rotation stress tests conducted using a fluoroscopy in the operation room. The patients with positive stress test and complete rupture in AITFL were indicated for the syndesmotic fixation. ${ }^{15}$

\section{Operative Methods}

The operation was performed by a surgeon. Patient was placed in semilateral position with bump under ipsilateral hip. A pneumatic tourniquet was applied at thigh level for free exam of syndesmotic stability. The operative approach was selected for longitudinal trans-fibular incision for combined fibular fracture and anterolateral incision over AITFL for isolated syndesmotic injury. We exposed the fracture site and anterolateral aspect of distal tibiofibular joint with extensor retinaculum and anterolateral ankle joint release. We should be careful about damage of superficial peroneal nerve. We confirmed ruptured AITFL with direct view. The fibular fracture was fixed with locking plate and screws, and the stability of syndesmosis using external rotation stress test with $\mathrm{C}$-arm and direct vision was tested. When the stress test was positive, we started syndesmotic fixation.

Firstly, it is necessary to reduce the syndesmosis precisely. The fibular pressed with thumb to attach to incisura fibularis of tibia. The fronts of the tibia and fibular should be kept flat.

For trans-syndesmotic screw fixation, a $4.5 \mathrm{~mm}$ fully threaded cortical screw was inserted from lateral cortex of fibular directed anteromedially at $30^{\circ}$ to the coronal plan and $2 \mathrm{~cm}$ proximal from the ankle joint, which was then removed 3 months postoperatively. Anatomical AITFL reconstruction was performed using suture anchors. The ruptured AITFL was not removed. Firstly, we inserted the $3.0 \mathrm{~mm}$ BioComposite SutureTak ${ }^{\circledR}$ (Arthrex, Florida, USA) into the fibular AITFL attachment site. Secondly, the retrieved No. 2 FiberWire suture was fixed at the tibial attachment site in the anatomical direction of AITFL using $3.5 \mathrm{~mm}$ BioComposite PushLock ${ }^{\circledR}$ (Arthrex, Florida, USA) (Figs 1 and 2). Postoperatively, the patients were immobilized about 4 weeks without weight bearing. After 4 weeks, ankle brace was applied and patients gradually returned to their previous activity with or without fracture.

\section{Clinical Evaluation}

The clinical results were determined using the AOFAS score and the OMAS at final follow-up. 


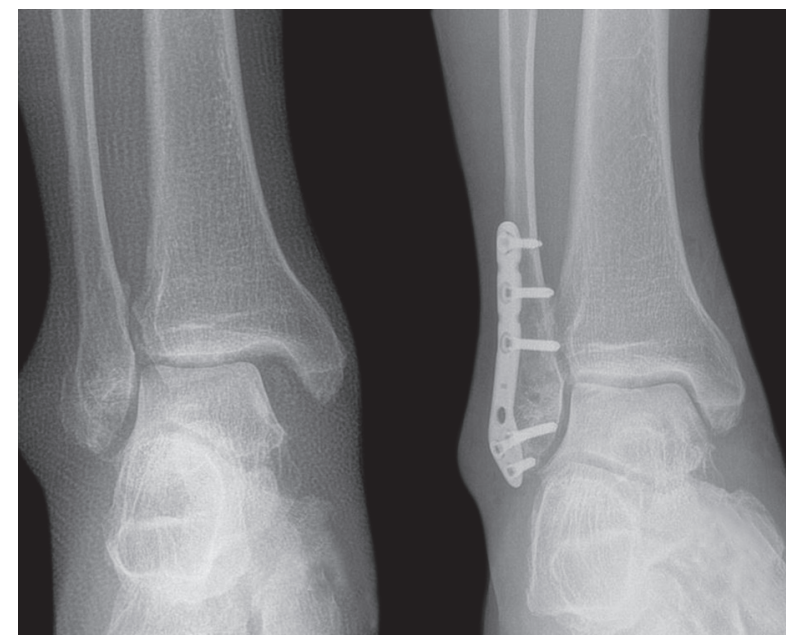

Fig. 1: A medial mortis widening that was suspected syndesmosis injury. We confirmed ruptured AITFL in the operation room. We reconstructed the syndesmosis with anchor sutures from fibular to tibia. The fractured fibula was fixed with anatomical locking plate and screws

\section{Radiologic Evaluations}

Tibiofibular overlapping, tibiofibular space, and tibiofibular overlapping ratio to the tibial width in anteroposterior ankle radiograph were measured at pre- and postoperative period. Computed tomography (CT) scan was conducted for the 25 (group I: 16, group II: 13) patients who could undergo it 1 month postoperatively. Gardner et al's ${ }^{16}$ and Phistkul et al's ${ }^{17}$ methods were used for decision of their anatomical reduction in the CT scan. We measured for quality of syndesmotic reduction by the anterior and posterior distance between the fibular and thee facets of the incisura at one-third and two-thirds position in the axial plane, which was placed $1 \mathrm{~cm}$ superior to the ankle joint. If the difference in distance between the anterior and posterior measurement was more than $2 \mathrm{~mm}$, it was considered as nonanatomical reduction (Fig. 3). ${ }^{16}$ The lengths of the anteroposterior and mediolateral axes were measured $1 \mathrm{~cm}$ superior to the ankle joint in an axial view. The normal ranges were -3 to $-1 \mathrm{~mm}$ and -4 to $0 \mathrm{~mm}$ respectively (Fig. 3). ${ }^{17}$

\section{Statistical Analysis}

Statistical Package for the Social Sciences (SPSS 16.0 for windows, Chicago, Illinois, USA) was used for statistical analyses. No normal distribution was observed; Mann-Whitney $U$ test was used to compare the final AOFAS, OMAS, and CT follow-up evaluation between groups I and II. Wilcoxon signed rank test was utilized to assess the preoperative and postoperative radiological evaluation. A post hoc power calculation was performed with G-power 3.0 (University Kiel, Germany). Statistical significance was accepted when $\mathrm{p}<0.05$.

\section{RESULTS}

\section{Clinical Results}

The final mean AOFAS of group I was 91.8 (82-100), group II was 91.5 (80-100), showing no significant difference between the groups $(\mathrm{p}=0.98)$. The mean OMAS of group I was 88.7 (65-100), and that of group II was 87.1 (60-100), showing no statistically significant difference between the groups $(\mathrm{p}=0.67)$ (Table 2$)$.
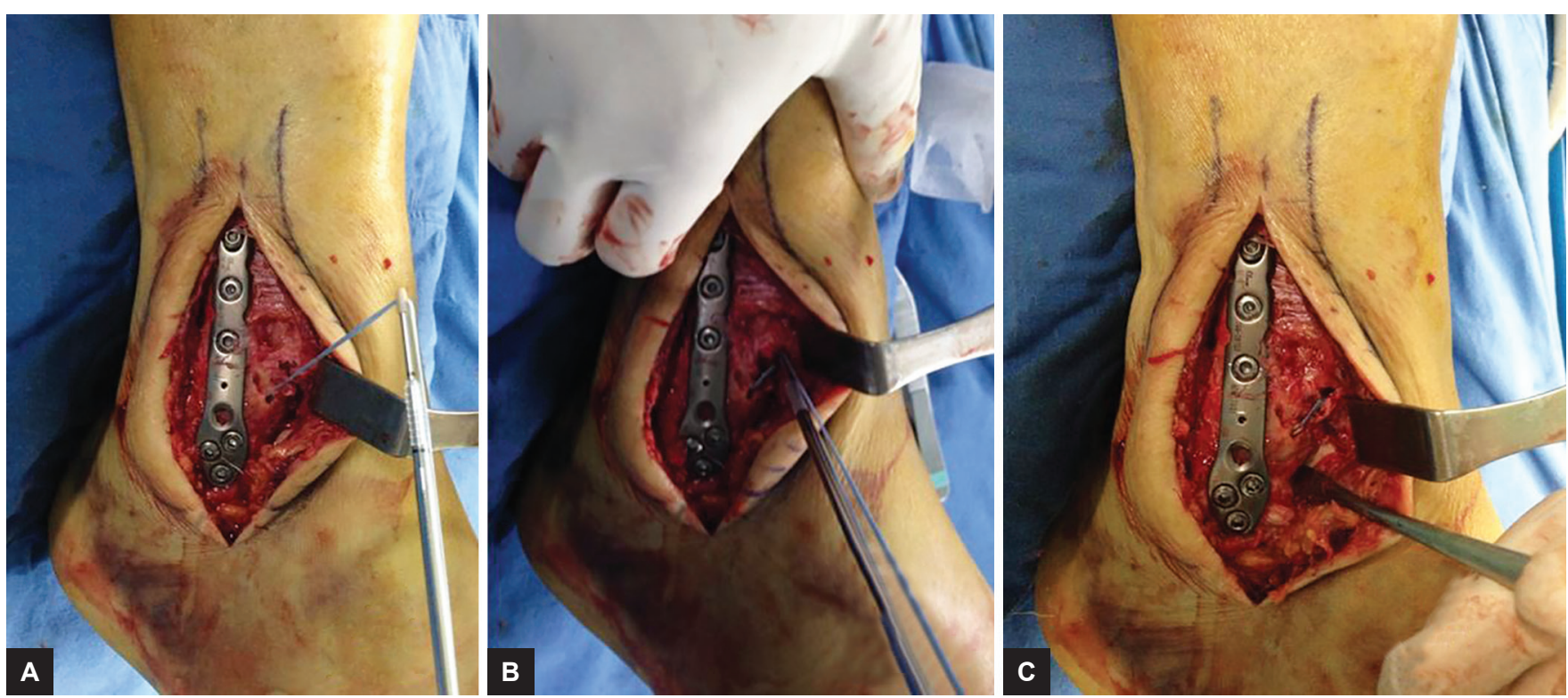

Figs 2 A to C: We usually fix the syndesmosis step by step. (A) Exposure of distal tibiofibular joint and AITFL complete rupture. The 3.0 $\mathrm{mm}$ BioComposite Suture Tak ${ }^{\circledR}$ (Arthrex, Florida, USA) was inserted into the fibular AITFL attachment site; (B) Second, the retrieved No. 2 FiberWire suture was fixed at the tibial attachment site in the anatomical direction of AITFL using 3.5 mm BioComposite PushLock; and $(C)$ We check again the stability of syndesmosis with freer elevator 


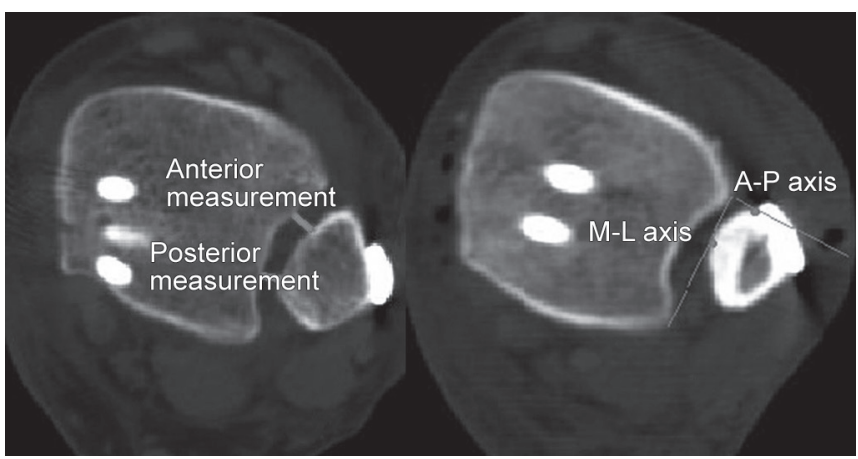

Figs 3A and B: (A) Gardner's method: Reduction quality was considered with axial CT scan measured $1 \mathrm{~cm}$ proximal to the ankle joint. If the difference in distance between the fibula and the anterior and posterior facets of the incisura was more than $2 \mathrm{~mm}$, it was considered as malreduction; (B) Phistkul's method: First, a reference line was drawn connecting the lateral-most points of the anterior and posterior tubercles of the incisura fibularis. Then, a second reference line was drawn from the anterior tubercle at a $90^{\circ}$ angle. Anteroposterior measurements were made from the reference line to the anterior-most point of the fibular cortex. Mediolateral measurements were made from the reference line to the medial-most point of the fibular cortex

\section{Simple Radiologic Test Results after the Operation}

In the simple radiologic images at the final follow-up, the mean tibiofibular overlapping distance in group I was 7.8 (5.15-12.59) $\mathrm{mm}$, and that in group II was 7.7 (6.01-10.31) $\mathrm{mm}$, confirming that both groups had reached the normal standard. The means tibiofibular space of group I was 3.3
(0.43-5.83) mm, and that of group II was 3.6 (2.45-4.67) $\mathrm{mm}$, confirming that both groups had been within the reduction standard. The tibiofibular overlapping ratio to the tibial width of group I was $41.6(0.11-0.53) \%$, and that of group II was $51.3(0.28-0.69) \%$, confirming that both groups had satisfied the reduction standard (Table 3).

\section{Computed tomography Scan Images 1 Month Postoperatively}

The mean anteroposterior length measured using Gardner's method in group I was -0.66 (-1.16 to -0.15$)$ $\mathrm{mm}$, and that in group II was $-0.85(-1.9$ to -0.12$) \mathrm{mm}$, confirming that both groups had been anatomically reduced (Table 4). The mean anteroposterior axis length measured using Phistkul's method in group I was -0.88 $(-2.07$ to 0.41$) \mathrm{mm}$ and that in group II was $-0.4(-1.21$ to $0) \mathrm{mm}$. There is a statistical difference between the two groups $(p<0.05)$. The mean mediolateral axis length of group I was 0.17 ( -0.74 to 0.61$) \mathrm{mm}$, and that of group II was -0.5 ( -1.49 to 0.83$) \mathrm{mm}$, confirming that both groups had been anatomically reduced (Table 5 ). We could not find the significant $\mathrm{CT}$ reduction measurement between both groups; however, post hoc power was relatively low.

\section{DISCUSSION}

Treatment methods for distal syndesmotic injury are still controversial. ${ }^{18}$ In the Kortekangas's study, ${ }^{13}$ it was reported that both the operative group using the cannulated screw

Table 2: Clinical outcomes after syndesmotic fixation

\begin{tabular}{lllll}
\hline & Cannulated screw (group I) & Suture anchor (group II) & $p$-value & $1-\beta$ error probability \\
\hline $\begin{array}{l}\text { American Orthopedic Foot and Ankle } \\
\text { Society }\end{array}$ & $91.8(82-100)$ SD 7.89 & $91.5(80-100)$ SD 8.11 & 0.98 & 0.064 \\
Olerud-Molander ankle score & $88.7(65-100)$ SD 12.90 & $87.1(60-100)$ SD 14.47 & 0.67 & 0.104 \\
\hline
\end{tabular}

SD: Standard deviation

Table 3: Plain radiographic assessment

\begin{tabular}{llc}
\hline & Cannulated screw (group I) & Suture anchor (group II) \\
\hline Preoperation (Min-Max) & & \\
TF Overlap (mm) & $3.83(2.76-4.91)$ & $3.72(2.64-4.79)$ \\
TF clear space (mm) & $6.56(6.02-7.09)$ & $5.56(5.12-6.01)$ \\
Overlap/fibular width (\%) & $25.26(18.42-32.10)$ & $25.64(18.52-32.76)$ \\
Final follow-up (Min-Max)) & & \\
TF overlap (mm) & $6.62(5.75-7.48)$ & $7.07(6.32-7.82)$ \\
TF clear space (mm) & $4.22(3.74-4.70)$ & $3.14(2.73-3.54)$ \\
Overlap/fibular width (\%) & $41.69(3.63-45.75)$ & $51.29(46.36-56.22)$ \\
\hline
\end{tabular}

Table 4: One month CT follow-up (Gardner's method)

\begin{tabular}{lllll}
\hline & Trans-syndesmotic screw (group I) & Suture anchors (group II) & $p$-value & $1-\beta$ error probability \\
\hline $\mathrm{N}$ & 16 & 31 & & \\
Anterior $(\mathrm{mm})$ & $3.11(2.70-3.51)$ SD 0.75 & $2.23(0.68-3.77)$ SD 0.99 & 0.097 & 0.834 \\
Posterior $(\mathrm{mm})$ & $3.77(3.24-4.29)$ SD 0.88 & $3.47(1.20-5.73)$ SD 1.23 & 0.541 & 0.181 \\
Anterior-Posterior $(\mathrm{mm})$ & $-0.66(-1.16--0.15)$ SD 0.69 & $-0.85(-1.9--0.12)$ SD 0.63 & 0.137 & 0.186 \\
\hline
\end{tabular}

SD: Standard deviation 
Anterior Inferior Tibiofibular Ligament Reconstruction with Anchor Sutures

\begin{tabular}{llllll}
\hline \multicolumn{5}{c}{ Table 5: One month CT follow-up (Phistkul's method) } & \\
\hline & Trans-syndesmotic screw (group I) & Suture anchors (group II) & p-value & $1-\beta$ error probability \\
\hline $\mathrm{N}$ & 16 & 13 & & \\
Anteroposterior axis $(\mathrm{mm})$ & $-0.88(-2.07$ to 0.41$)$ SD 0.38 & $-0.4(-1.21$ to 0$)$ SD 0.71 & 0.115 & 0.711 \\
Mediolateral axis $(\mathrm{mm})$ & $0.17(-0.74$ to 0.61$)$ SD 0.39 & $-0.5(-1.49$ to 0.83$)$ SD 0.89 & 0.237 & 0.816 \\
\hline
\end{tabular}

and the non-operative group did not show significant differences in the midterm follow-up resutls. However, other many studies show that operative treatments had been needed because the unstable distal syndesmosis could have resulted in a persistent ankle pain or in degenerative changes. ${ }^{5,7,18-21}$

For ideal distal syndesmotic fixation, a tension that allows stable fixation during the ligament recovery and early joint mobilization should be maintained while not interfering with with the normal biomechanical movements. ${ }^{15}$

The conventional screw fixation method has the limitations of changing the normal biomechanical movements of the distal syndesmosis and of requiring removal. ${ }^{3,22}$ According to gait biomechanical studies, the syndesmosis allowed movement of $1 \mathrm{~mm}$ on the medial lateral axis and externally rotated 2 degrees with ankle dorsiflexion. According to Needleman et $\mathrm{al}_{,}{ }^{17}$ the $4.5 \mathrm{~mm}$ conventional screw fixation at the syndesmosis restricted the anteroposterior and external motion of the talus. Thus, the screw should be removed to prevent the screw breaking when bearing weight. ${ }^{3,417}$ Recently, a flexible fixation like suture button for syndesmotic injury has been used, and favorable results have been reported instead of rigid screw fixation..$^{10,18}$ Soin et $\mathrm{al}^{22}$ reported that there was no significant difference between the suture-button and screw fixation groups. However, Forsythe et $\mathrm{al}^{11}$ reported that one suture-button fixation with a transverse plane could not maintain anatomical reduction. In particular, it was difficult to maintain the tension when the fibular had externally rotated force in sagittal plane. In the biomechanical study conducted by Teramoto et $\mathrm{al}_{1}{ }^{23}$ the conventional screw fixation was too strong, and the conventional single or double suturebutton fixation method showed that it could maintain an anatomical reduction of syndesmosis with load bearing. However, suture-button fixation at the transverse plane could not stabilize the tibiofibular syndesmosis during external rotation force They recommended the anatomical suture button fixation directed from the posterior cortex of the fibula to the anterolateral edge of the tibia to be most similar to the intact specimen. In this study, the anatomical reconstruction of the anteroinferior tibiofibular ligament was performed using suture anchors instead of suture-button material. When using Teramoto's anatomical suture-button fixation technique ${ }^{24}$ it is difficult to make the hole, and there is a peroneal tendon irritation problem. Our technique makes it easy to ensure the stability of the AITFL, which has more anatomical fixation via the upper surface of the AITFL. ${ }^{25}$

In a cadaver study by Seitz et $\mathrm{al}^{26}$ the mean pullout strength in cannulated screw fixation was $82 \mathrm{lbs}$, and in suture-button fixation, $49 \mathrm{lbs}$ in suture-button fixation, and in a study by Barber et $\mathrm{al}^{2}$ the pullout strength was $44 \mathrm{lbs}$. Although the pullout strength did not reach that of the aforementioned two methods, the strength was good enough to endure the force applied to the distal syndesmosis during the normal joint movements. When external rotation and adduction were conducted in the operative room with ankle dorsiflexion, findings of indicated that stability was maintained. We needed temporary anatomical stability of the syndesmosis for the fibrotic healing of the AITFL and the syndesmosis.

Malreduction of the distal syndesmosis can occur in $0-16 \%$ of the cases using cannulated screw fixation. ${ }^{27}$ In this study, both screw fixation and suture anchor fixation were performed by an experienced clinician with direct vision, and we obtained accurate reduction. Less experienced physicians may have an increased risk of performing malreduction with screw fixation. Less experienced physicians may have an increased risk of performing malreduction with screw fixation. Cherney et $\mathrm{al}_{,}^{8}$ reported the $1 \mathrm{~mm}$ excessive compression and 5 -degree external rotation after indirect reduction with ball-point pelvic reduction forceps. We directly reduced the syndesmosis by free hand compression without compression forceps; this method avoided excessive compression of the syndesmosis. We do not need removal due to the bio-composite anchor. We allowed weight bearing at 4 weeks, and the results indicated less postoperative stiffness because we did not need to wait until removal of the screw. The broken screw tips remained inside of the patient's tibia; it was not related to clinical outcomes.

The limitations of this study included that the followup period of the suture anchor patient group was comparatively short and that the sample sizes of the CT scans in both groups were too small to accurately determine the anatomical reductions. Retrospectively, the post-hoc power was also weak. Further studies with a longer follow-up period and a larger sample size may be necessary in the future. 


\section{CONCLUSION}

When reductions were performed in syndesmotic injury patients through the fixation methods involving the use of the conventional cannulated screws and suture anchors, no significant difference was observed between the methods in terms of the clinical and plain radiologic results. But suture anchor method showed good results in CT follow-up. Through the simple suture anchor method, however, anatomical reduction was realized, and the normal anatomical biomechanical movements were recovered, without ankyloses and additional operation..$^{28}$

\section{REFERENCES}

1. Amendola A. Controversies in diagnosis and management of syndesmosis injuries of the ankle. Foot Ankle Int 1992 Jan;13(1):44-50.

2. Barber FA, Herbert MA, Richards DP. Sutures and suture anchors: update 2003. Arthroscopy 2003 Nov;19(9):985-990.

3. Bell DP, Wong MK. Syndesmotic screw fixation in Weber C ankle injuries - should the screw be removed before weight bearing? Injury 2006 Sep;37(9):891-898.

4. Beumer A, Campo MM, Niesing R, Day J, Kleinrensink GJ, Swierstra BA. Screw fixation of the syndesmosis: a cadaver model comparing stainless steel and titanium screws and three and four cortical fixation. Injury 2005 Jan;36(1):60-64.

5. Beumer A, Valstar ER, Garling EH, Niesing R, Ginai AZ, Ranstam J, Swierstra BA. Effects of ligament sectioning on the kinematics of the distal tibiofibular syndesmosis: a radiostereometric study of 10 cadaveric specimens based on presumed trauma mechanisms with suggestions for treatment. Acta Orthop 2006 Jun;77(3):531-540.

6. Beumer A, Valstar ER, Garling EH, van Leeuwen WJ, Sikma W, Niesing R, Ranstam J, Swierstra BA. External rotation stress imaging in syndesmotic injuries of the ankle: comparison of lateral radiography and radiostereometry in a cadaveric model. Acta Orthop Scand 2003 Apr;74(2):201-205.

7. Canale ST, Beaty JH. Campbell's operative orthopaedics. Expert consult premium edition, enhanced online features. Elsevier Health Sciences; 2012.

8. Cherney SM, Haynes JA, Spraggs-Hughes AG, McAndrew CM, Ricci WM, Gardner MJ. In vivo syndesmotic overcompression after fixation of ankle fractures with a syndesmotic injury. J Orthop Trauma 2015 Sep;29(9):414-419.

9. Chun KY, Choi YS, Lee SH, Kim JS, Young KW, Jeong MS, Kim DJ. Deltoid ligament and tibiofibular syndesmosis injury in chronic lateral ankle instability: magnetic resonance imaging evaluation at 3T and comparison with arthroscopy. Korean J Radiol 2015 Sep-Oct;16(5):1096-1103.

10. Cottom JM, Hyer CF, Philbin TM, Berlet GC. Treatment of syndesmotic disruptions with the arthrex tightrope ${ }^{\mathrm{TM}}$ : a report of 25 cases. Foot Ankle Int 2008 Aug;29(8):773-780.

11. Forsythe K, Freedman KB, Stover MD, Patwardhan AG. Comparison of a novel FiberWire-button construct versus metallic screw fixation in a syndesmotic injury model. Foot Ankle Int 2008 Jan;29(1):49-54.
12. Gardner MJ, Demetrakopoulos D, Briggs SM, Helfet DL, Lorich DG. Malreduction of the tibiofibular syndesmosis in ankle fractures. Foot Ankle Int 2006 Oct;27(10):788-792.

13. Pakarinen HJ1, Flinkkilä TE, Ohtonen PP, Hyvönen $\mathrm{PH}$, Lakovaara MT, Leppilahti JI, Ristiniemi JY. Syndesmotic fixation in supination-external rotation ankle fractures: a prospective randomized study. Foot Ankle Int 2011 Dec;32(12): 1103-1109.

14. Lee SH, Kim ES, Lee YK, Yeo ED, Oh SR. Arthroscopic syndesmotic repair: technical tip. Foot Ankle Int 2015 Feb;36(2): 229-231.

15. Marqueen T, Owen J, Nicandri G, Wayne J, Carr J. Comparison of the syndesmotic staple to the transsyndesmotic screw: a biomechanical study. Foot Ankle Int 2005;26(3):224-230.

16. Miller R, Weinhold P, Dahners L. Comparison of tricortical screw fixation versus a modified suture construct for fixation of ankle syndesmosis injury: a biomechanical study. J Orthop Trauma 1999 Jan;13(1):39-42.

17. Needleman RL, Skrade DA, Stiehl JB. Effect of the syndesmotic screw on ankle motion. Foot Ankle Int 1989 Aug;10(1):17-24.

18. van den Bekerom MP, Raven EE. Current concepts review: operative techniques for stabilizing the distal tibiofibular syndesmosis. Foot Ankle Int 2007 Dec;28(12):1302-1308.

19. Oh JK, Wang JM, Roh KJ, Yun YH, Kim DJ, Jeong H. Diagnostic landmarks of ankle syndesmosis separation measured on standard ankle anterior-posterior radiographs of normal Korean adults. J Korean Orthop Assoc 1998 Oct;33(5): 1263-1266.

20. Phisitkul P, Ebinger T, Goetz J, Vaseenon T, Marsh JL. Forceps reduction of the syndesmosis in rotational ankle fractures. J Bone Joint Surg 2012 Dec;94(24):2256-2261.

21. Zalavras C, Thordarson D. Ankle syndesmotic injury. J Am Acad Orthop Surg 2007 Jun;15(6):330-339.

22. Soin SP, Knight TA, Dinah AF, Mears SC, Swierstra BA, Belkoff SM. Suture-button versus screw fixation in a syndesmosis rupture model: a biomechanical comparison. Foot Ankle Int 2009 Apr;30(4):346-352.

23. Teramoto A, Suzuki D, Kamiya T, Chikenji T, Watanabe K, Yamashita T. Comparison of different fixation methods of the suture-button implant for tibiofibular syndesmosis injuries. Am J Sports Med 2011 Oct;39(10):2226-2232.

24. Teramoto A, Kura H, Uchiyama E, Suzuki D, Yamashita T. Three-dimensional analysis of ankle instability after tibiofibular syndesmosis injuries: a biomechanical experimental study. Am J Sports Med 2008 Feb;36(2):348-352.

25. Nelson OA. Examination and repair of the AITFL in transmalleolar fractures. J Orthop Trauma 2006 Oct;20(9):637-643.

26. Seitz WH, Bachner EJ, Abram LJ, Postak P, Polando G, Brooks DB, Greenwald AS. Repair of the tibiofibular syndesmosis with a flexible implant. J Orthop Trauma 1991 Mar;5(1):78-82.

27. Weening B, Bhandari M. Predictors of functional outcome following transsyndesmotic screw fixation of ankle fractures. J Orthop Trauma 2005 Feb;19(2):102-108.

28. Thornes B, Shannon F, Guiney A-M, Hession P, Masterson E. Suture-button syndesmosis fixation: accelerated rehabilitation and improved outcomes. Clinical Orthop Relat Res 2005 Feb;431:207-212. 